\title{
La guerra fría cultural en el Tercer Mundo: el Congreso por la Libertad de la Cultura en México e India
}

\section{The Cultural Cold War in the Third World: The Congress for Cultural Freedom in Mexico and India}

\author{
Daniel Kent Carrasco* \\ (D) https://orcid.org/0000-0002-9381-3654 \\ Instituto de Investigaciones Históricas, UNAM \\ danielkentca@gmail.com
}

Resumen: El presente artículo desarrolla una historia comparativa de las trayectorias de las sedes del Congreso por la Libertad de la Cultura en México e India durante la década de 1950. El planteamiento central de este escrito es que las actividades promovidas desde ambas sedes desempeñaron un papel central en el establecimiento de una cultura liberal de elite articulada en torno a la denuncia del peligro que el totalitarismo de izquierda representaba para el futuro de ambos países y, por extensión, para el resto del Tercer Mundo. En una época en la que la batalla por las ideas definía no sólo las coordenadas geopolíticas globales, sino también la orientación subjetiva y las aspiraciones de los intelectuales cosmopolitas del Tercer Mundo, esta cultura de elite fue

* Doctor en Historia del Pensamiento Político Contemporáneo, King’s College, Londres. Líneas de investigación: historia global, historia intelectual de India y México, historia de los contactos entre India y México durante el siglo xx.

cómo cITAR: Kent Carrasco, D. (2021). La guerra fría cultural en el Tercer Mundo: el Congreso por la Libertad de la Cultura en México e India. Secuencia (111), e1931. DoI: https://doi.org/10.18234/secuencia. v0i111.1931

c) 98

Esta obra está protegida bajo una Licencia Creative Commons Atribución-NoComercial 4.0 Internacional. 
central para la consolidación de un nuevo consenso intelectual definido por el anticomunismo y la defensa del liberalismo como único horizonte político.

Palabras clave: anticomunismo; liberalismo de la guerra fría; historia global del Tercer Mundo; modernización; guerra fría cultural.

Abstract: This article undertakes a comparative history of the trajectories in the headquarters of the Congress for the Freedom of Culture in Mexico and India during the 1950s. The central point of this writing is that the activities promoted in both places played a central role in the establishment of an elite liberal culture centered around the denouncement of the danger posed by leftwing totalitarianism for the future of both countries and, by extension, the rest of the Third World. At a time when the battle for ideas defined not only global geopolitical coordinates but also the subjective orientation and aspirations of Third World cosmopolitan intellectuals, this elite culture was central to the consolidation of a new intellectual consensus defined by anti-communism and the defense of liberalism as the only political horizon.

Keywords: anticommunism; cold war liberalism; global history of the Third World; modernization; cultural cold war.

Recibido: 12 de enero de 2021 Aceptado: 24 de mayo de 2021

Publicado: 1 de octubre de 2021

$\mathrm{E}$ n septiembre de 1963, Ivan Katz, miembro del secretariado del Congreso por la Libertad de la Cultura (CLC), localizado en París, mandó una carta a Prabhakar Padhye, director de la oficina local del cLc en Delhi. En su misiva, Katz animaba a Padhye a visitar la sede del Congreso en la ciudad de México con la finalidad de instaurar un canal de comunicación directa entre los representantes del Congreso en ambos países del Tercer Mundo. Para Katz, las sedes del CLC en Delhi y la capital mexicana eran comparables no sólo en términos organizativos, sino también, y sobre todo, en función del papel que ambas desempeñaban en relación con la vida cultural, intelectual y política de 
sus respectivas regiones: Asia y Latinoamérica. ${ }^{1}$ Por esto, el contacto directo entre sus miembros sería de enorme importancia para la cruzada intercontinental del Congreso. La visita de Padhye a México nunca llegó a concretarse. Sin embargo, la sugerencia de Katz forma parte de una serie de intercambios, encuentros y paralelismos gestados entre las sedes del CLC en México e India durante los últimos años de la década de 1950 y la primera mitad de la de 1960. Durante aquellos años, entre ambas sedes fluyeron noticias, recomendaciones y materiales impresos marcados por el impulso internacionalista del Congreso por la Libertad de la Cultura, organización central para la ofensiva cultural global de Estados Unidos durante los inicios de la guerra fría.

Fundado en Berlín en junio de 1950 en respuesta al Consejo Mundial por la Paz, impulsado desde la Unión Soviética, el CLC se constituyó como una organización internacional enfocada en la denuncia del totalitarismo -un término que por aquellas épocas tomó gran importancia en los debates intelectuales internacionales a partir del impacto de la obra de autores como Hannah Arendt y Karl Popper-, la defensa de la libertad intelectual y cultural, la promoción de la democracia liberal y el impulso anticomunista que alimentaba al naciente imperialismo estadunidense. A lo largo de casi una década y media, el CLC fundó numerosas revistas en distintas lenguas -suajili, español, japonés, ruso, árabe y chino-, financió proyectos editoriales y exhibiciones, brindó becas a artistas y escritores, y organizó una nutrida red de eventos en todos los continentes. Entre las figuras que recibieron apoyo del Congreso se cuentan George Orwell, Albert Camus, Isaiah Berlin, V. S. Naipaul, Buddhadeva Bose, Thomas Mann, Bertrand Russell, Raymond Aron, Alfonso Reyes, Jorge Luis Borges, Octavio Paz y Juan Rulfo. Desde un inicio, el Congreso por la Libertad de la Cultura fue financiado con fondos que la Agencia Central de Inteligencia (CIA) canalizaba a través de distintas instancias no gubernamentales y fundaciones filantrópicas entre las que resaltaba la Fundación Ford. Esto fue mantenido en secreto hasta 1967, año en el que una serie de reportajes publicados en la revista estadunidense Rampart's y el periódico The New York Times hicieron público el origen de los fondos, dinamitando el prestigio del CLC a nivel global.

${ }^{1}$ Carta de Ivan Katz a Prabhakar Padhye, 19 de septiembre de 1963. International Association for Cultural Freedom Records (en adelante IACFR), caja 181, exp. 10. Special Collections. The University of Chicago Library. En inglés en el original. Traducción del autor. Todas las traducciones de aquí en adelante son del autor. 
Retratado por un historiador como el "equivalente cultural-intelectual de la economía política del Plan Marshall”, el cLC inicialmente buscó cimentar una "gran coalición" de intelectuales afincados en las capitales culturales del Atlántico Norte en defensa de una renovación de la perspectiva liberal que permitiera articular una crítica intercontinental al comunismo y los peligros del totalitarismo (Scott-Smith, 2001, pp. 2-6). En 1955, en reacción a la difusión global del proyecto de solidaridad no alineada impulsado desde la Conferencia Afro-Asiática de Bandung celebrada en abril de aquel año, el CLC llevó a cabo un esfuerzo consciente de expandir sus actividades y rango de influencia en el Tercer Mundo (Burke, 2016). Como resultado de esto, durante los años siguientes se gestó una amplia red de intercambios entre figuras y organizaciones asociadas al CLC y sus defensores en Latinoamérica, Asia y África, animada por la celebración de congresos, seminarios, publicaciones y correspondencias en distintas lenguas y todos los continentes del mundo.

Desde un inicio, el Congreso por la Libertad de la Cultura se dio a la tarea de unificar a diversos círculos de intelectuales cosmopolitas en defensa de los valores del Mundo Libre. Moldeada a partir de las ruinas de las guerras ideológicas que azotaron a la Europa de entreguerras, la imagen del Mundo Libre proponía una alternativa para los proyectos enfrentados del socialismo y el fascismo a partir de dos reclamos interconectados. En primer lugar, sus defensores promovían un internacionalismo anticomunista encabezado por Estados Unidos como la única forma aceptable de política para el mundo de la posguerra. Por otro lado, defendía los valores liberales como remedio a la expansión del totalitarismo comunista. En este sentido, el cLC desempeñó un papel central en la redefinición de las coordenadas ideológicas y discursivas del liberalismo durante los primeros años de la guerra fría. En una influyente exposición de este nuevo "liberalismo de la guerra fría", Arthur M. Schlesinger -autor del bestseller internacional El centro vital. Una politica de la libertad (1949) y defensor del CLC desde sus inicios ${ }^{2}$ - afirmaba que el liberalismo debía convertirse en una nueva "fe militante" para el mundo de la posguerra (Schlesinger jr., 2017). En años subsecuentes, otros influyentes colaboradores del CLC como Edward Shils, Daniel Bell e Isaiah Berlin complementaron la lectura militante de Schlesinger jr. con un rotundo rechazo del pensamiento ideológico y el entusiasmo utópico para dar paso a una nueva agenda cos-

${ }^{2}$ Schlesinger jr. estuvo presente y activo en la primera sesión del cLc. Congress Pour la Liberte de la Culture. Séance du 30 Novembre 1950. IACFR, caja 56, exp. 7. 
mopolita de debate antirrevolucionario a la que se sumarían intelectuales de países como India y México, contribuyendo a la cristalización de una nueva agenda cultural, artística y académica marcada por la defensa del liberalismo antiautoritario de la guerra fría. ${ }^{3}$

El impacto de las actividades del Congreso por la Libertad de la Cultura en el Atlántico Norte ha sido ampliamente documentado y discutido en las últimas décadas como parte central de la llamada "guerra fría cultural" (Coleman, 1989; Saunders, 2001; Scott Smith, 2000, 2001, 2002; Rubin, 2012). La historia del CLC ha dado pie a una perspectiva descentrada que entrelaza perspectivas locales, regionales y transcontinentales en el afán de comprender los vínculos de la organización con diversas agendas y actores regados alrededor del mundo en las primeras décadas de la posguerra. En años recientes, esta perspectiva ha alimentado una batería de fascinantes estudios que han enriquecido nuestra comprensión de su funcionamiento en Latinoamérica (Alburquerque, 2011; Glondys, 2018; Iber, 2015; Jannello, 2012, 2014; Ruiz Galbete, 2018) y, en menor medida, en Asia y África (Holt, 2013; Pullin, 2011). Mención especial merece el trabajo de Roland Burke (2016), quien apunta hacia la importancia de entender el funcionamiento del CLC en el amplio terreno del Tercer Mundo, un espacio simbólico y geopolítico que representó el escenario central de la guerra fría global (Westad, 2005). En el presente ensayo, analizaremos los vínculos, paralelismos y diferencias entre las sedes del Congreso en India y México con miras a contribuir al estudio transnacional -y transcontinental- de las redes de intercambio cultural e intelectual que durante las décadas tempranas de la guerra fría vincularon a regiones lejanas del Tercer Mundo a través de la lógica marcada por las compulsiones ideológicas y geopolíticas que encauzaron el nuevo imperialismo estadunidense y su lucha por la contención del comunismo a lo largo y ancho del planeta. A partir del análisis de los materiales contenidos en los archivos de la Asociación Internacional por la Libertad de la Cultura, guardados en la Universidad de Chicago, este escrito plantea que las actividades promovidas desde el Congreso por la Libertad de la Cultura en México e India desempeñaron un papel central en el establecimiento de una cultura liberal de elite articulada en torno a la denuncia del peligro que el totalitarismo de izquierda

${ }^{3}$ Sobre el posicionamiento de estos autores respecto a los ideales rectores del cLC, véase Bell (1960), Berlin (1990) y Shils (1990). Para más sobre los contornos del "liberalismo de la guerra fría”, véase Anderson (2011). 
representaba para el futuro de ambos países y, por extensión, para el resto del Tercer Mundo. En una época en la que la batalla por las ideas definía no sólo las coordenadas geopolíticas globales, sino también la orientación subjetiva y las aspiraciones de los intelectuales cosmopolitas del Tercer Mundo, esta cultura de elite fue central para la consolidación de un nuevo consenso intelectual transnacional definido por la centralidad del anticomunismo y la defensa del liberalismo como único horizonte político. A partir de estas premisas, este artículo pretende contribuir a los recientes debates historiográficos que, en diálogo con el trabajo de historiadores como Westad, buscan discutir los procesos de la guerra fría en perspectiva global. Al mismo tiempo, pretende hacer un comentario comparativo de los entornos de la alta cultura en India y México en las décadas de 1950 y 1960 con miras a ir trazando paralelismos y puntos de encuentro que permitan pensar en una historia comparativa de más largo alcance entre ambos países.

\section{FRONTERAS LEJANAS DEL MUNDO LIBRE}

En una de las reuniones iniciales del CLC, celebrada en noviembre de 1950, James Burnham -miembro de la delegación estadunidense y célebre promotor de la doctrina de la "contención del comunismo"- anunció tener un "amigo indio" que había expresado "un gran interés y deseaba entrar en contacto con el Congreso". " Es muy probable que este "amigo indio" haya sido Minoo Masani, socialista indio de inclinaciones anticomunistas con quien Burnham había estado en contacto desde mediados de la década de $1940 .{ }^{5}$ En efecto, poco después de esta reunión inicial, en enero de 1951, Masani encabezó la creación del Comité Indio por la Libertad de la Cultura (Indian Committee for Cultural Freedom, ICCF), organización que rápidamente se puso

${ }^{4}$ Congress Pour la Liberte de la Culture. Séance du 27 Novembre 1950. IACFR, caja 56, exp. 7.

${ }^{5}$ Fundador del Partido Socialista del Congreso en 1934, Masani tuvo una larga e impresionante carrera política: fue miembro de la Corte Suprema de Bombay, del Consejo General del Congreso Indio de Sindicatos (All-India Trade Union Congress), del Comité General del Congreso Nacional Indio (1936-1938), alcalde de Bombay (1943-1944), miembro de la Asamblea Constituyente de la República de India y embajador de India en Brasil (1947-1948). Desde mediados de la década de 1940, Masani desarrolló una animada relación epistolar con James Burnham, cuyas cartas pueden ser consultadas en los archivos de la Institución Hoover, en Stanford California. https://oac.cdlib.org/findaid/ark:/13030/tf0p3000sz/entire_text/ 
en contacto con la oficina central del CLC en París para expresar el deseo de sus allegados de encabezar una "reunión de escritores" y otros miembros de la intelligentsia de "los países libres" de Asia para reafirmar la defensa de la "libertad individual" y "los procesos democráticos" en un continente que se descolonizaba a pasos agigantados. En su tono y propuesta -en su carta se habla con miedo del "comunismo mundial" y del "lavado de cerebros" encabezado por sus defensores-, el ICCF hacía claro eco de la paranoia antitotalitaria compartida por muchos miembros del CLC y planteaba a los intelectuales liberales de India como sus aliados de este último en su cruzada internacional. ${ }^{6}$ La respuesta de París no pudo haber sido más entusiasta. Para principios de febrero, el Comité Ejecutivo del CLC anunció que había decidido enviar a India una delegación de sus miembros europeos más renombrados -incluyendo a Raymond Aron, Ignazio Silone, Stephen Spender y Salvador de Madariaga- y se aseguraba que el evento contaría también con el apoyo de su sede estadunidense -encabezada por Burnham, Sydney Hook y Arthur Koestler-. ${ }^{7}$ La iniciativa del ICCF pronto se confirmó como la primera actividad apoyada por el cLC en suelo no europeo.

Los esfuerzos encabezados por Masani culminaron con la celebración del Congreso Indio por la Libertad de la Cultura, inaugurado el 28 de marzo de 1951 en Bombay. El evento contó con la presencia de intelectuales del Atlántico Norte, como Madariaga, Spender y Norman Thomas, e importantes representantes de la vida cultural y política de India como B. R. Ambedkar, Ashoka Mehta y Jayaprakash Narayan. Durante sus sesiones, se leyeron mensajes de apoyo de figuras como Karl Jaspers, Eleanor Roosevelt, John Dos Passos y Arthur Schlesinger jr. A pesar de la pretensión de limitarse al ámbito neutral de la cultura, el evento estuvo marcado por una clara retórica anticomunista y antisoviética que entraba en conflicto con la política neutralista defendida por el primer ministro Jawaharlal Nehru. ${ }^{8}$ Era evidente, declaró Denis de Rougemont desde el podio en Bombay, que el comunismo representaba "el tipo más peligroso de totalitarismo" del mundo y que Estados Unidos, si bien no encarnaba "al bien o la verdad", representaba la única espe-

${ }^{6}$ Indian Committee for Cultural Freedom. A Working Paper prepared in connection with a preliminary meeting of writers and others from the countries of South, South-East and East Asia. IACFR, caja 173, exp. 5.

${ }^{7}$ Réunion du Comité Exécutif. Versailles, 9-11 de febrero de 1951. IACFR, caja 56, exp. 8.

${ }^{8}$ Sobre la posición de Nehru respecto al No Alineamiento, véase Nanda (1998); Nayudu (2015). 
ranza internacional para la libertad. ${ }^{9}$ Por su parte, el propio Masani recalcó la necesidad que tenían los intelectuales del Tercer Mundo de superar la "USAfobia" (USAphobia) y declararse abiertos defensores del Mundo Libre (Indian Congress for Cultural Freedom, 1951, pp. 246-264). En su reporte final del evento, Masani celebró el éxito del evento y reiteró que era natural que, después de su fundación en Berlín en 1950, la segunda reunión internacional del CLC hubiera tomado lugar en una ciudad india. La misma lucha que "estaba tomando lugar en torno al alma de Berlín y Alemania", declaró, "estaba tomando lugar también en India”. En una reiteración retórica del imaginario geográfico de la guerra fría, Masani anunciaba triunfalmente que Bombay representaba el equivalente asiático de la capital alemana y describía a India como el último puesto de avanzada en las "fronteras de la libertad". ${ }^{10}$

La filial india del CLC siguió un camino ascendente de consolidación durante el siguiente lustro. En mayo de 1952 Minoo Masani fue integrado al Comité Ejecutivo Internacional de la organización ${ }^{11}$ y entre 1951 y 1956 a las oficinas de Bombay se sumaron nuevas sedes en Delhi y Calcuta, así como presencia en numerosas localidades menores del país. Durante 1954, desde la oficina de Delhi-comandada por el periodista Prabhakar Padhye-se organizó la Segunda Conferencia Asiática por la Libertad de la Cultura, celebrada en la ciudad de Rangún, en febrero de 1955, y que reunió a decenas de intelectuales del Atlántico Norte y Asia (Pullin, 2011; Lewis, 2019). Celebrada siete meses antes de la conferencia "El futuro de la Libertad", organizada por el CLC en Milán en septiembre de 1955, comúnmente reconocida como el momento de consolidación de la presencia global del Congreso (Burke, 2016; Scott Smith, 2001), la conferencia de Rangún -dominada por intelectuales indios- confirmó la centralidad de la filial india para la misión intercontinental del cLC. Durante aquellos años las actividades del ICCF incluyeron a importantes figuras del mundo cultural y político de la época como los poetas Nissim Ezekiel y Buddhadeva Bose, el novelista Sachchidananda Vatsyayana, el editor Eric Da Costa, el pedagogo Sampurnanad y el sociólogo Rajni Kothari. En 1960, el Comité Ejecutivo del clc en París premió el tesón de Masani y sus compañeros nombrando a la sede de Delhi como la oficina regional del Congreso para

\footnotetext{
${ }_{9}$ Denis de Rougemont. The Indian Congress for Cultural Freedom. IACFR, caja 173, exp. 7.

${ }_{10}$ M. R. Masani. The Indian Congress for Cultural Freedom. IACFR, caja 173, exp. 7.

${ }^{11}$ Repórt on the Reunion Du Comité Executif. Paris, 31 de mayo de 1952. IACFr, caja 56, exp. 9.
} 
todo Asia, dotando a sus actividades de un alcance continental y un reconocimiento global que ninguna otra sede del mundo poseía. ${ }^{12}$

Por otro lado, en noviembre de 1950, el mismo Burnham puso sobre la mesa del recientemente creado cLc la posibilidad de celebrar un "congreso de algún tipo" en América Latina -"quizás en México"- que pudiera servir para cimentar la presencia de la organización en la región. ${ }^{13}$ Esta propuesta fue muy bien recibida en las filas del CLC. El escritor francés David Rousset celebró la posibilidad de contribuir a la urgente tarea de fomentar la existencia de una izquierda anticomunista en Latinoamérica. ${ }^{14}$ De manera similar a lo que sucedió en India, "la gran importancia intelectual y artística" de México fue reconocida por los altos mandos del Congreso muy tempranamente, lo que contribuyó a que el país se convirtiera en una pieza central de su estrategia internacional. Finalmente, en los últimos meses de 1953, se estableció en México una "Agencia distribuidora de publicaciones del Congreso" dedicada principalmente a la distribución de la revista Cuadernos del Congreso por la Libertad de la Cultura, editada desde París por el valenciano Julián Gorkin. ${ }^{15}$ Situada en la librería Ariel en el 91 de la calle Donceles, esta agencia de distribución sirvió como núcleo original de la Asociación Mexicana por la Libertad de la Cultura (AMLC), creada en agosto de 1954 y encabezada por el editor y periodista Rodrigo García Treviño.

Durante los siguientes años, la AMLC pasó de ser un mero centro de distribución de materiales impresos a tener presencia en varios estados de la república y contacto con decenas de intelectuales y artistas del país. La primera iniciativa de la Asociación consistió en organizar una exposición de pintura destinada a terminar con la "dictadura" ejercida por los "pintores comunistas Diego Rivera [y] David Alfaro Siqueiros" en el ámbito de las artes plásticas, la cual fue celebrada en enero de 1955 en las Galerías Excélsior. ${ }^{16} \mathrm{~A}$ partir de aquel momento, la AMLC extendió sus contactos en círculos universitarios - de la Universidad Nacional, donde contó con la colaboración del rec-

12 "Minutes of the Annual General Meeting”, ICCF Bullettin, junio de 1960. IACFR, caja 175, exp. 7.

${ }_{13}$ Congress Pour la Liberte de la Culture. Séance du 27 Novembre 1950. IACFR, caja 56, exp. 7; Réunion du 10 Févreir 1951 - 10 h. 30. IACFR, caja 56, exp. 8.

${ }^{14}$ Réunion du 10 Févreir 1951 - 10 h. 30. IACFr, caja 56, exp. 8.

${ }_{15}$ Carta de Nicolas Nabokov a Mauricio Magdaleno, 28 de septiembre de 1953. IACFR, caja 222 , exp. 5.

${ }^{16}$ Activities of the Latin American Committees of the Congress for Cultural Freedom During the Last Six Months of 1954. IACFR, caja 57a, exp. 1. 
tor Nabor Carrillo y el director de la Facultad de Filosofía y Letras, Salvador Azuela, y el Instituto Politécnico Nacional- y oficiales -contó con la participación activa del funcionario del Instituto Nacional de Bellas Artes, Salvador Pineda, el senador Pedro de Alba, y despertó el interés de importantes figuras como el regente de la ciudad de México, Ernesto P. Uruchurtu-. ${ }^{17} \mathrm{Al}$ mismo tiempo, la Asociación sirvió de plataforma para la actividad de importantes artistas, académicos e intelectuales de la época, entre los que resaltan Rufino Tamayo, Alfonso Reyes, Manuel Rodríguez Lozano, Octavio Paz, Mauricio Magdaleno, Felipe Cossío del Pomar, Margarita Michelena, Rodolfo Usigli, Daniel Cosío Villegas y Víctor Urquidi. Cuatro años después de su creación, la Asociación contaba con nuevas oficinas en Puebla, Veracruz y Saltillo, y reportaba un total de 100 "socios regulares" y "relaciones e influencia" con más de 1000 intelectuales avecindados en la capital. ${ }^{18}$

El momento cúspide de la historia del CLC en México tuvo lugar con la celebración en septiembre de 1956 de la Conferencia Interamericana del Congreso por la Libertad de la Cultura. La conferencia, que contó con la participación de delegados e invitados de España, Estados Unidos, Colombia, Argentina, Bolivia, Ecuador, Canadá, El Salvador, Puerto Rico, Cuba, Brasil, Costa Rica, Uruguay y Chile, tenía la intención de ser una "confrontación de intelectuales Norte y Latinoamericanos" que resaltara su unidad de propósito, más allá de las fronteras, en defensa de los ideales de la libertad y en contra del imperio de la ideología. ${ }^{19}$ Entre los presentes se encontraban importantes intelectuales de distintos rincones del continente, como Germán Arciniegas, Benjamín Carrión, Rómulo Gallegos, John Dos Passos, Ralph Waldo Ellison, Frank Tannenbaum, Norman Thomas, Roger Baldwin y Luis Alberto Sánchez. ${ }^{20}$ Celebrada en el Auditorio Nacional, la conferencia de 1956 tuvo un importante impacto mediático y despertó el interés de las altas esferas de la política mexicana, incluyendo el del presidente Ruiz Cortines. Cauto de caer en "fáciles optimismos", Julián Gorkin apuntó que la Conferencia significó

\footnotetext{
${ }_{17}$ Julián Gorkin, The Congress for Cultural Freedom in Latin America, 1953. IACFR, caja 215, exp. 1; carta de Víctor Alba a Francois Bondy, 2 de junio de 1953. IACFR, caja 214, exp. 5.

${ }_{18}$ Carta de Rodrigo García Treviño a John Hunt, 21 de marzo de 1958. IACFr, caja 215, exp. 3.

${ }^{19}$ Carta de Julián Gorkin a don Rodrigo García Treviño, 13 de enero de 1956. IACFR, caja 93, exp. 5.

${ }^{20}$ Programa Conferencia Interamericana del Congreso por la Libertad de la Cultura. IACFR, caja 237, exp. 10.
} 
un triunfo para la estrategia internacional del CLC ya que había logrado ofrecer "una prueba rotunda, ante toda la opinión latinoamericana, del espíritu de libertad y de independencia de nuestro Congreso". ${ }^{21}$

Para inicios de 1958, los dirigentes del CLC en París tenían amplios motivos para celebrar la actividad de sus filiales en México e India. En una nota escrita en enero de aquel año, John Hunt, agente de la cia y secretario del CLC, reconocía que tanto la AMLC como el ICCF representaban piezas clave en la estrategia de expansión global de la organización y afirmaba que sus oficinas servían activamente como plataformas para nuevas iniciativas en países como Ceilán, Birmania, Brasil y Colombia. ${ }^{22}$

\section{PALADINES DE OCCIDENTE EN EL TERCER MUNDO}

Tanto en México como en India, el CLC alimentó el surgimiento de un nuevo frente de intelectuales de elite, que se erigieron como defensores de los parámetros del liberalismo de la guerra fría y la agenda de la modernización. Haciendo eco de la defensa de la premisa liberal que promovía la autonomía de los intelectuales respecto al poder y las agendas políticas como valor supremo, los defensores del cLc planteaban que el mundo de las ideas y la cultura debía erigirse como la piedra de toque de una sociedad verdaderamente libre. En este sentido, se adherían a los planteamientos de autores como Lionel Trilling quien, en su famosa obra de 1950, proponía que la creación de una nueva "imaginación liberal" -que definiera tanto los contornos de la práctica política como el horizonte de la actividad artística- era la labor más urgente y acuciante de la posguerra (Trilling, 2008). Al mismo tiempo, las iniciativas del CLC nacían de la convicción de que la ideología era el peor enemigo de la libertad intelectual y debía ser desterrada para abrir paso al surgimiento de una auténtica racionalidad en la arena pública. El liderazgo en estos procesos recaía en la figura del intelectual de elite quien debía encabezar, en palabras

${ }^{21}$ Informe de Julián Gorkin sobre la Conferencia de México, 24 de octubre de 1956. IACFR, caja 237, exp. 12. Esta conferencia representó el segundo evento internacional del CLC en Latinoamérica. El primero había tenido lugar en Santiago, en junio de 1954. Al respecto, véase Jannello (2012).

${ }^{22}$ Activities of the National Committees of the Congress for Cultural Freedom, 1957; $y$ Comité Excecutif International, 18-19 de enero de 1958. Brief by John C. Hunt. IACFR, caja 58, exp. 4. 
de Edward Shils (1958), la clausura "del entusiasmo ideológico" que había definido las coordenadas de la política internacional en las primeras décadas del siglo xx y pugnar por el establecimiento de una nueva perspectiva para el análisis social basada en la crítica al "fanatismo y la posesión ideológica".

En el caso de India, las actividades del CLC marcaron un momento importante en la cristalización de una nueva figura del intelectual poscolonial, definida por las compulsiones del liberalismo de la guerra fría y las pugnas políticas internas del país asiático. En un texto publicado en 1955 en Foreign Affairs, Minoo Masani (1955) afirmaba que los intelectuales en Asia poseían un poder superior al de "la aristocracia terrateniente o los capitalistas" y tenían la capacidad de "hacer y deshacer gobiernos". En breves, gracias a sus vínculos con Occidente y a su capacidad de representar a los sectores modernos y cosmopolitas del continente, para Masani los intelectuales representaban a la verdadera "clase dirigente" en Asia. A partir de esta elitista fantasía, los seguidores del Congreso en Asia imaginaban a los intelectuales como los principales puentes entre los valores tradicionales de sus respectivas sociedades y las posibilidades de la modernización política y económica de la región. En este sentido, se adherían a los argumentos de Shils (1958, p. 454), quien durante aquellos años planteaba que los intelectuales de los "nuevos países" de Asia y África debían renunciar al sueño de la revolución para encabezar una reinterpretación de los patrones tradicionales capaz de contribuir a la modernización de sus sociedades.

De los seguidores del CLC en India, nadie encarnaba esta visión mejor que Jayaprakash Narayan. Viejo líder sindicalista, ideólogo marxista y fundador del Partido Socialista del Congreso (1934), durante la década de 1950 Narayan se volcó hacia un proyecto que unía los ideales del socialismo y el pensamiento de Gandhi y enfatizaba la importancia del individualismo, la autonomía respecto al Estado y la pugna por la creación de una "política civil" (lok niti) que reflejaba los planteamientos "post-ideológicos" de los seguidores del CLC (Kent Carrasco, 2016, 2020). Para los impulsores del Congreso en el Atlántico Norte, la participación de Narayan -un respetado líder popular visto por muchos como el más probable sucesor de Nehru en el puesto de primer ministro y una figura capaz de traducir los ideales del liberalismo de la guerra fría al lenguaje político de India-era de enorme importancia. Durante la década de 1950, el socialista Gandhiano fue nombrado Presidente de Honor del Consejo -puesto que compartía con importantes estandartes de la cultura occidental como Karl Jaspers, John Dewey y Bertrand Russell-y sus obras tra- 
ducidas a numerosas lenguas, incluyendo el español, y distribuidas ampliamente a través de las redes del cLc en el Tercer Mundo.

En el caso de México, las actividades del Congreso fueron encabezadas por dos fervientes anticomunistas: el español Julián Gorkin y el mexicano Rodrigo García Treviño. Nacido en 1901, Gorkin inició su carrera política en el Partido Comunista y, más tarde, editó La Batalla, periódico del Partido Obrero de Unificación Marxista (poum). Huyendo de España en 1939, el valenciano llegó por primera vez a México en abril de 1940, donde compartiría departamento con el bolchevique desencantado Víctor Serge (Amat, 2016, pp. 29-39; Iber, 2015, p. 19). En México, Gorkin se erigió como un virulento promotor de la causa antitotalitaria y anticomunista, que defendería desde su trinchera de editor y empresario cultural. ${ }^{23}$ El valenciano, que dejó México en 1947 y se desempeñó durante años como editor de la revista Cuadernos del Congreso por la Libertad de la Cultura y nexo entre la oficina de París del CLC y los países de Latinoamérica, compartía con Masani la convicción de que la intelligentsia "democrática y antitotalitaria" debía erigirse como un baluarte en la defensa del Mundo Libre y encabezar la causa liberal en la guerra fría cultural..$^{24}$ Su contraparte en México, Rodrigo García Treviño, era un antiguo colaborador de Trotsky y editor de la obra de Marx en México (Rivera Mir, 2018). Activo comentarista de la política mexicana y ferviente antiestalinista, el periodista se definía a sí mismo como un lector atento del senador estadunidense McCarthy y un declarado enemigo de los "estalinistas criollos" encabezados por Vicente Lombardo Toledano. ${ }^{25}$

Más allá de las similares perspectivas de sus principales defensores, es importante entender las actividades del cLc en México e India en función de las posturas ideológicas y geopolíticas de los gobiernos de ambos países en el horizonte de la temprana guerra fría. Por un lado, en India el cLc desde un inicio tuvo que superar la desconfianza que sus actividades despertaron en importantes círculos gubernamentales que incluían al propio Jawaharlal Nehru. Importante impulsor del Movimiento de los Países No Alineados y

${ }^{23}$ Durante su paso por México (1940-1948), Gorkin fundó las editoriales Ediciones Libres y Ediciones Quetzal, desde donde publicaría títulos como Caníbales políticos (Hitler y Stalin en España) y la traducción del libro de Serge Hitler contra Stalin.

${ }^{24}$ Report by Julian Gorkin non the Congress for Cultural Freedom in Latin America. IACFR, caja 57, exp. 5.

${ }^{25}$ Carta de Rodrigo García Treviño a Julián Gorkin, 6 de octubre de 1953. IACFR, caja 222, exp. 5. 
estandarte del impulso de la descolonización de Asia y África, el primer ministro indio (1947-1964) recelaba profundamente de cualquier crítica lanzada en contra de la política exterior neutralista de India, en especial cuando estas contaban con el apoyo de agentes internacionales. Al mismo tiempo, y a pesar de su declarado afán por defender la neutralidad, Nehru se definía a sí mismo como un socialista y desconfiaba profundamente de Estados Unidos cuyos políticos, escribía en 1945, "pensaban que podían resolver cualquier problema a través del dinero y las armas" e ignoraban "el fuerte resentimiento de los pueblos de Asia en contra de las imposiciones" imperialistas. ${ }^{26}$ Desde el inicio, Nehru vio con claridad que el cLC representaba los ideales de la política de la contención del comunismo estadunidense, una sospecha que era compartida por otros miembros de la elite política india que asociaban la labor de agencias filantrópicas, como la Fundación Rockefeller y la Fundación Ford, con una nueva forma de imperialismo cultural (McGarr, 2013, p. 15; Roosevelt, 1954, p. 90). Esto no pasó desapercibido para los altos mandos del CLC, quienes muy tempranamente identificaron los "gestos hostiles" de Nehru como la principal amenaza para la libertad de la cultura en India y Asia. ${ }^{27}$

En contraste, en México las actividades del Congreso se combinaron armónicamente con el anticomunismo que durante las décadas de 1940 y 1950 permeó las actitudes de las elites políticas y culturales del país. Durante aquellos años, que marcaron el auge del modelo de desarrollo estabilizador o "milagro mexicano", se vivió el eclipse de la coalición de fuerzas e ideas que habían impulsado el proyecto nacional popular impulsado por Lázaro Cárdenas y la consolidación de un modelo de desarrollo que benefició a los sectores urbanos y capitalistas del país. Sobre las bases de la estabilidad social generada por los primeros gobiernos posrevolucionarios, durante esta década se impulsó un nuevo orden socioeconómico marcado por la alianza entre el gobierno y el capital privado nacional y extranjero, y el anhelo creciente de modernización. En términos geopolíticos, durante estos años la agenda del gobierno mexicano favoreció un marcado acercamiento con Estados Unidos que transformó radicalmente los hábitos y expectativas de la población urbana de México, sobre todo en la capital (Moreno, 2004). Estos cambios socioculturales coincidieron con la gradual aceptación del anticomunismo por parte

${ }^{26}$ Carta de Jawaharlal Nehru a G. D. Birla, 21 de mayo de 1954. G. D. Birla Papers, Nehru Memorial Museum and Library, citada en McGarr (2013, p. 23).

${ }^{27}$ Francois Bondy, “Bombay Congress”, 11 de abril de 1951. IACFR, caja 173, exp. 7. 
del gobierno de México, cuyos líderes se adhirieron a la estrategia geopolítica de la guerra fría encabezada por Estados Unidos. El presidente Manuel Ávila Camacho (1940-1946) cooperó con los Aliados en la segunda guerra mundial, mientras que Miguel Alemán (1946-1952) y Adolfo Ruiz Cortines (1952-1958) fueron receptivos a la política exterior del vecino del norte y entusiastas de la inversión estadunidense en México. En este nuevo horizonte marcado por la creciente presencia del capital y el poder geopolítico estadunidense, el comunismo comenzó a ser visto como una amenaza para el orden social en México y la modernización del país. En palabras de Miguel Alemán, el comunismo era incompatible con "el mexicanismo" (Medina, 1979, pp. 176-180).

$\mathrm{Al}$ margen de estos dilemas políticos, en ambos países los defensores de la libertad de la cultura abogaron por la necesidad de reafirmar la integración de sus respectivas sociedades en el caudal de la cultura occidental. En el caso de México y Latinoamérica, región descrita en las páginas de Cuadernos como la "prolongación espiritual" de Europa y "saturad(a) de cultura occidental” (Santos, 1956, p. 19), esta era una tarea sencilla. Después de todo, los intelectuales mexicanos y latinoamericanos "reconoc[ían] que le deb[ía] n mucho -si no es que todo- a la cultura europea" y entusiastamente aceptaban "la defensa de Europa y sus libertades [como] la defensa de una posesión común". ${ }^{28}$ Estas afirmaciones hallaban un eco en los anhelos de los sectores cosmopolitas de las elites culturales de México, cuyos integrantes enfáticamente afirmaban su pertenencia a la civilización occidental y se regían por la premisa articulada por el joven Octavio Paz, quien en 1950 afirmaba que los mexicanos se encontraban "por primera vez en [su] historia" en condiciones de ser "contemporáneos de todos los hombres" (Paz, 1993, p. 317). Al tiempo que continuaban defendiendo las principales premisas del nacionalismo revolucionario de décadas anteriores, durante la temprana de posguerra las elites intelectuales de México promovieron una definición cosmopolita de la cultura mexicana y persiguieron fervientemente el sueño de integración con el mainstream de la cultura occidental siguiendo los patrones intelectuales definidos desde las capitales culturales del Atlántico Norte (Cohn, 2005; Franco, 2002).

Del otro lado del mundo, y a pesar de los entusiastas anhelos de Minoo Masani, India representaba un reto desconcertante para los defensores de la

${ }^{28}$ Report by Julian Gorkin on the Congress for Cultural Freedom in Latin America. IACFR, caja 57, exp. 5. 
libertad de la cultura fincados en el Atlántico Norte. A diferencia de los mexicanos asociados con el CLC -como el modernista Octavio Paz y el erudito de la antigüedad griega Alfonso Reyes- los intelectuales indios aparecían como herederos de una "compleja" y "constantemente desconcertante [...] tradición cultural". Durante una visita al país, celebrada en 1954, el secretario general del CLC, Nicolas Nabokov, declaró que para "un occidental, como yo" India representaba "una experiencia para la cual no existen criterios de comparación" ${ }^{29}$ Esta extrañeza, compartida por numerosos representantes occidentales del CLC en India, estaba enmarcada por añejas ideas sobre el lugar ocupado por India en la narrativa universalista del ascenso de la modernidad -desde el siglo XVIII, pensadores occidentales como G. W. F. Hegel, Karl Marx y Max Weber habían contribuido a fijar una imagen de India como una tierra de "atraso" y "despotismo", ajena al cambio y resistente a la "revolución política" (Anderson, 2010, pp. 13-28, 208-212; Hegel, 1956, pp. 142 y 154; Metcalfe, 1997, pp. 66-112) - y por fantasías orientalistas que identificaban a India como "la madre [...] de los ideales y las creaciones de Europa" (Indian Congress for Cultural Freedom, 1951, p. 160). Estas visiones se combinaban con ansiedades contemporáneas sobre el papel geopolítico del país en la arena de la guerra fría. A diferencia de México, cómodamente situado en la esfera de influencia de Estados Unidos, India, situada en la colindancia del Mundo Libre, la URSS y la República Popular China, era un país constantemente a merced de la expansión comunista.

Al margen de las divergentes condiciones generadas por las diferentes presiones geopolíticas y posiciones ideológicas en cada país, tanto en India como en México el CLC sirvió como una importante plataforma para el impulso de agendas intelectuales vinculadas al ideal de la modernización durante los primeros años de la década de 1960. Acuñado en la década de 1930 por autores como Robert Redfield, durante la temprana posguerra el término "modernización" comenzó a ser utilizado ampliamente por autores interesados en el estudio de Asia, África y Latinoamérica -un grupo que incluía a figuras de enorme peso como Edward Shils, Talcott Parsons y Clifford Geertz- para describir un modelo de desarrollo lineal y universal definido por nociones de progreso y evolución social marcadamente eurocéntricas mediante el cual se pensaba que todas las sociedades serían capaces de transitar de un estado de atraso definido por el predominio de la "tradición" hacia una nueva etapa

${ }^{29}$ Nicholas Nabokov, Report of my trip to Southeast Asia and Japan. IACFR, caja 57a, exp. 2. 
marcada por la llegada de la "modernidad". Esta última era concebida como el resultado de la superación del subdesarrollo y el atraso, y asociada con las virtudes y el horizonte del capitalismo industrial y la democracia liberal. Esta nueva teoría, que cimentó una nueva ideología transnacional, emergía de la convicción de que las naciones del Atlántico Norte, y en especial Estados Unidos de América, mostraban el camino del progreso y el desarrollo para las naciones del Tercer Mundo (Gilman, 2003; Latham, 2003). En complemento, el ideal de la modernización fue entendido por las elites de los países de Asia, África y Latinoamérica como el camino para "alcanzar" al Occidente. En 1955, por ejemplo, Nehru declaraba que India debía lograr "en diez o quince" años lo que "Europa" había hecho en "cien o ciento cincuenta" (Nehru, 2002 citado en Chakrabarty, 2005), mientras que Julius Nyerere, presidente de Tanzania, eligió titular su autobiografía Nosotros debemos correr mientras ellos caminan (We must run while they walk). A medida que la confrontación de la guerra fría incrementaba la inestabilidad del Tercer Mundo, el prospecto de la modernización justificó la intervención estadunidense y la consolidación de agendas desarrollistas abiertamente anticomunistas apoyadas por las elites de Latinoamérica, Asia y África. En palabras del ideólogo W. W. Rostow, autor de Etapas del crecimiento económico: un manifiesto no comunista (1960), la modernización formaría la base para una "nueva relación [...] entre la mitad norte y la mitad sur del Mundo Libre" y aseguraría el acercamiento entre los "hombres libres" del mundo, tanto los pobres como los ricos. ${ }^{30}$ En este sentido, queda claro que la modernización evolucionó como complementó al liberalismo postutópico y antitotalitario de los primeros años de la guerra fría y dotó un marco analítico capaz de integrar al Tercer Mundo en el seno del proyecto del Mundo Libre.

La agenda de la modernización fue impulsada desde el cLC en México e India a través del contacto de sus sedes con un nuevo grupo de intelectuales: los académicos. Hacia finales de la década de 1950, las actividades de las sedes del Congreso en ambos países complementaron su enfoque en las artes -la pintura y la literatura principalmente-con un acercamiento con importantes círculos académicos. En India, esta labor fue encabezada por Ashoka Mehta - un viejo socio de Masani y Narayan y miembro del Partido Socialista

30 Memorandum, Rostow to Theodore Sorenson, 16 de marzo de 1961. National Security Files, box 325, "Rostow, Foreign Aid, 3/16/61-3/18/61", John F. Kennedy Library, Boston, Massachusetts, citado en Latham (2003, p. 16). 
del Congreso- quien encabezó numerosos seminarios, grupos de discusión y comisiones de trabajo financiados por el CLC. Bajo su tutela, desde las oficinas indias del Congreso se organizaron charlas de figuras internacionales como el sociólogo estadunidense del MIT Myron Weiner, el historiador del liberalismo estadunidense Louis P. Hartz y otros académicos de la London School of Economics y las universidades de Ohio, Kansas, Melbourne y Harvard, ${ }^{31}$ así como de académicos indios como S. C. Dube (1958), importante estudioso de las dinámicas de cambio social en la India rural y Rajni Kothari, uno de los politólogos más influyentes de la segunda mitad del siglo en aquel país. En el plano internacional, Mehta desempeñó un papel de gran importancia en las actividades del CLC en el Tercer Mundo, al formar parte del comité organizador, junto con Edward Shils, de la conferencia internacional "La democracia en los nuevos Estados", celebrada bajo el auspicio del Congreso en la isla griega de Rodas en 1958 y concebida como la continuación de la importante conferencia de Milán de 1955. En India, los esfuerzos de Mehta culminaron con la creación de la Comisión de los Intelectuales, un grupo conformado por funcionarios del gobierno indio y académicos encabezados por Mehta y Shils que durante la década de 1960 se reunió para discutir sobre el futuro de la sociedad india y encaminarla hacia la "transición" hacia la modernidad. ${ }^{32} \mathrm{~A}$ pesar de su corta existencia (1964-1966), los trabajos de esta Comisión de los Intelectuales marcaron una nueva etapa en la evolución del intelectual liberal indio de la guerra fría, una figura que había transitado de ser el representante simbólico de las aspiraciones de su país a representar el principal motor de su modernización.

En México, la agenda de modernización adoptada por los principales defensores del CLC involucró a importantes figuras de la vida académica del país. Para Keith Botsford y Luis Mercier Vega, enviados del CLC en México durante la década de 1960, uno de los principales obstáculos de la modernización de México residía en el sistema de educación superior del país. Estos observadores opinaban que este último estaba marcado por la mediocridad y enfocado meramente en la "auto-reproducción" de sus propios cuadros y

\footnotetext{
${ }^{31}$ Indian Committee for Cultural Freedom, Calcutta Centre, Report of activities during 1966. IACFR, caja 179, exp. 1.

${ }^{32}$ Intellectual's Commission on the Problem of Open Community, Proceedings of the meeting held in the offices of the Congress for Cultural Freedom on 11 October 1964; The Intellectuals Commission, 5 Hailey Road, New Delhi, Report of the Meeting held on 7 January 1965. IACFR, caja 182 , exp. 3 .
} 
estructuras. La ausencia de incentivos para la investigación seria y el rigor académico, junto con la politización de la vida universitaria convertían al sistema universitario mexicano en una plataforma para la perpetuación de una “clase privilegiada" en lugar del mecanismo de movilidad social central para la modernización. Como resultado de este "inmovilismo espiritual", los intelectuales emergidos de las universidades de México eran incapaces de superar sus atavismos tradicionales y competir en el "mercado libre de las ideas" como sus contrapartes en el Atlántico Norte. ${ }^{33}$ Para contrarrestar la percibida mediocridad de las instituciones universitarias del país, las actividades del CLC comenzaron a enfocarse crecientemente en los círculos académicos de México. En octubre de 1964 se celebraron en la ciudad de México una serie de reuniones del Comité de Planeación Latinoamericano del CLC, integrado por académicos como Ornaldo Fals-Borla de la Universidad Nacional de Colombia, Víctor Urquidi de El Colegio de México, Peter Heintz de la Flacso-Chile, Emir Rodríguez Monegal del Instituto de Profesores Artigas en Montevideo, Ramón Di Zubiria de la Universidad de los Andes en Bogotá, Jaime García Terrés de la UNAM, Germán Carrera Damas de la Universidad Central de Venezuela, y Juan Marichal de la Universidad de Harvard. ${ }^{34}$ En asociación con Stephen Graubard, editor de la prestigiosa revista Daedalus, agentes del CLC, como Mercier Vega, encabezaron una serie de seminarios sobre distintos temas de interés académico, incluyendo el papel de Latinoamérica en el orden mundial..$^{35}$ Siguiendo la línea dictada por los defensores de la teoría de la modernización, estas reuniones ayudaron a establecer una esfera de discusión alejada de los extremos ideológicos y las "tomas de posición abstractas" para México y toda América Latina defendidas por figuras cercanas al marxismo, como el sociólogo Pablo González Casanova. ${ }^{36}$ Este esfuerzo, paralelo al de la Comisión de los Intelectuales en India, alimentó la creación del Instituto Latino Americano de Relaciones Internacionales (ILARI) en Ginebra en 1966, organización que contó con la participación de intelectuales como

${ }^{33}$ Draft Memorandum on Latin America, Keith Botsford and Luis Mercier Vega, 14 de mayo de 1963. IACFR, caja 561, exp. 13.

${ }_{34}$ Planning Committee Latin America-Mexico, 28-31 de octubre de 1964. IACFR, caja 215, exp. 9.

${ }^{35}$ Agradezco al(la) lector(a) anónimo(a) del primer borrador de este artículo el haberme introducido a estos detalles de la historia del involucramiento del CLC con las elites académicas mexicanas de la década de 1960 .

${ }^{36}$ Luis Mercier Vega to Luis Guillermo Piazza, 21 de enero de 1965. IACFR, caja 215, exp. 11; A proposed Latin American Quarterly. IACFR, caja 215, exp. 10. 
Gino Germani, Seymour Lipset, Albert O. Hirschman y Daniel Cosío Villegas, quienes pretendieron enfocarse en el estudio del "mundo real en el que la gente de América Latina vive cotidianamente" para contribuir al crecimiento de un debate público objetivo, libre de posturas extremistas y conducente a la modernización de las sociedades de Latinoamérica. ${ }^{37}$

En breve, del estudio de las funciones de las sedes del Congreso por la Libertad de la Cultura en India y México, la conclusión emerge de que en ambos países el involucramiento de importantes intelectuales locales sirvió para promover los valores, intereses y modelos de desarrollo impulsados desde el Atlántico Norte, a través de la defensa de una esfera de actividad cultural e intelectual claramente alineada con los planteamientos liberales y modernizadores que durante la temprana guerra fría fueron asociados con el constructo de Occidente (Latham, 1997).

\section{EL PROBLEMA DEL IMPERIALISMO}

A pesar de estas coincidencias, existieron importantes contradicciones que marcaron la trayectoria del CLC en México e India. En términos ideológicos, la más importante surgió de las concepciones encontradas en torno al imperialismo esgrimidas por los defensores de la libertad de la cultura en estos países. Como hemos visto, el CLC era entendido por sus promotores como una organización representativa de los valores del Mundo Libre y enfrentada a la expansión del totalitarismo comunista que, a los ojos de sus líderes, aparecía como una forma de imperialismo más peligrosa y dañina que la dominación colonial que las potencias europeas habían ejercido en África, Asia y Latinoamérica en siglos anteriores. A pesar de su entusiasmo respecto a los valores liberales y modernizadores defendidos desde Occidente, las elites cosmopolitas de India y México tenían serias dudas respecto a este planteamiento. Herederos de una sensibilidad política forjada en el fuego del anticolonialismo, los intelectuales liberales de India tenían dificultades para desvincular el concepto de totalitarismo del recuerdo reciente del imperialismo británico y para asociar el liderazgo del Atlántico Norte con la democracia. Por su lado, y a pesar de sus inclinaciones cosmopolitas, importantes sectores de las elites

${ }^{37}$ Functions and Programs of the Instituto Latinoamericano de Relaciones Internacionales. IACFR, caja 215, exp. 10. 
intelectuales de México de la temprana posguerra abiertamente rechazaban el imperialismo estadunidense y aceptaban tácitamente los planteamientos del "nacionalismo revolucionario" del régimen del Partido Revolucionario Institucional (PRI).

Al mismo tiempo, ambos países ocupaban un lugar ambiguo en la emergente geografía bipolar creada por el conflicto de la guerra fría. En el caso de México, esto quedó claramente de manifiesto durante la década de 1950 con las reacciones del régimen y la sociedad respecto al golpe de Estado orquestado por la ciA en Guatemala en 1954 y el alzamiento revolucionario encabezado por Fidel Castro en Cuba en 1959. La caída del gobierno de Jacobo Árbenz, ocurrido en un momento de creciente cercanía entre los gobiernos de Dwight Eisenhower y Adolfo Ruiz Cortines, revivió a importantes sectores de la izquierda antiimperialista en México, acallados durante la década de 1940 y reforzó la imagen del país como foco importante del pensamiento revolucionario en Latinoamérica (Loaeza, 2016). Pocos años después, la caída de Fulgencio Batista a manos de un grupo de guerrilleros que durante años habían vivido libremente en México agudizó la tensión entre la agenda de acercamiento entre el gobierno de Adolfo López Mateos y Estados Unidos y la necesidad del régimen del PRI de reafirmar sus credenciales revolucionarias en el continente (Keller, 2015). Esto no impidió, sin embargo, que durante la década de 1960 México se volviera un aliado cercano de Estados Unidos, un importante receptor de ayuda internacional y, más allá de la retórica, un miembro más de los países del Mundo Libre. Del otro lado del mundo, India, un país que recibió muy poca atención por parte del gobierno estadunidense durante la década de 1940, emergía como un jugador de enorme importancia en la geopolítica del sur de Asia (Chaudhuri, 2013). En contraste con el gobierno del PRI -claramente alineado con Estados Unidos desde el inicio de la guerra fría- el régimen del Congreso Nacional Indio logró armonizar las buenas relaciones comerciales y diplomáticas con la Unión Soviética con el acercamiento estratégico respecto a Estados Unidos. Durante la segunda mitad de la década de 1950, el comercio entre India y la URSS se multiplicó por siete al tiempo que el país del sur de Asia se convertía en uno de los principales destinos de la ayuda internacional estadunidense (McGarr, 2013, pp. 3 y 35). En los años siguientes, y a pesar del entusiasmo de Nehru por el modelo soviético y el prospecto de la solidaridad no alineada, India se acercó significativamente al campo del Mundo Libre después de la guerra sino-india de 
1962 que devastó los cimientos del panasianismo y pulverizó las esperanzas del tercermundismo en Asia (Sen, 2017, pp. 379-469).

Estas fricciones y ambigüedades causaron perplejidad y preocupación entre los defensores de la libertad de la cultura en India y México. Para Gorkin, a quien el golpe de Estado en Guatemala sorprendió en medio de una gira por Latinoamérica, era difícil explicar "la casi unánime reacción de los elementos democráticos" del continente "a favor de Árbenz". El valenciano reaccionó con alarma ante la postura de algunos miembros del CLC en México según quienes el "Departamento de Estado [de Estados Unidos], junto con la United Fruit Company" eran los verdaderos artífices de la caída de Árbenz, figura reconocida como el líder de una "revolución agraria y democrática" coartada por la intromisión de la ciA. Perplejo, Gorkin atribuía el rechazo del "imperialismo yankee" extendido entre los intelectuales mexicanos a la facilidad con que estos últimos eran engañados por la "propaganda comunista, a pesar de estar convencidos de lo contrario". Para el valenciano, el "antiimperialismo sistemático y miope" de las elites intelectuales de México representaba un importante obstáculo para el anticomunismo en Latinoamérica y volvía al país un nodo central en la cruzada internacional en defensa de la libertad de la cultura en el mundo ${ }^{38}$ En el caso de India, el antiimperialismo anticolonial de las elites nacionalistas encabezadas por Jawharlal Nehru se complementaba con una desconfianza generalizada en contra de "todo lo remotamente" relacionado con o "patrocinado desde Occidente". Durante una visita realizada a India a finales de 1955, Nicholas Nabokov constató que, a pesar del entusiasmo de las elites intelectuales respecto al Mundo Libre, en las calles de Delhi era perceptible la abierta admiración popular por la Unión Soviética y la China comunista. Su preocupación se tornó en alarma cuando observó la "parafernalia que adornaba" el escritorio de Nehru, que incluía "fotografías de Mao y Chou En Lai [sic.]", "retratos de líderes soviéticos enmarcados en plata" y una "horrenda 'troika' labrada en madera blanca acompañada de un enorme oso". 39

Esta tensión entre el anticomunismo del cLC y el antiimperialismo imperante en India y México -marcado por el colonialismo británico en el caso del país del sur de Asia y por el recelo antiestadunidense en el caso del país latinoamericano- quedó de manifiesto con las protestas organizadas

${ }_{38}$ Report on Latin America by Julián Gorkin. IACFR, caja 57a, exp. 1. Las cursivas son mías. 
desde la plataforma del CLC en contra de la intervención soviética en Hungría en octubre de 1956. En el caso de India, el Congreso sirvió como base para una enérgica manifestación encabezada por Jayaprakash Narayan en apoyo a la "lucha revolucionaria" del pueblo de Hungría "en contra de la dominación Rusa”. ${ }^{40}$ Esta postura abiertamente antisoviética contrastó fuertemente con la actitud del gobierno de Nehru, quien fue extremadamente cauto y, durante varias semanas, declinó a declararse respecto a la situación en Hungría (Reid, 1981, pp. 147-148). Para Narayan, la postura de Nehru ante Hungría resultaba vergonzosa y contrastaba dramáticamente con el abierto apoyo otorgado por el primer ministro hacia la nacionalización del canal de Suez encabezada por Nasser en Egipto pocos meses antes. ${ }^{41}$ La protesta encabezada por el ICCF contra la postura del gobierno indio aumentó hasta que Nehru finalmente se declaró en contra de la presencia soviética en Hungría el 19 de noviembre, cambio que fue celebrado por sus miembros como un triunfo para los defensores de la libertad de la cultura. ${ }^{42}$ En contraste a lo sucedido en India, en México los defensores del CLC se vieron incapaces de dirigir una reacción similar ante los acontecimientos en Hungría. En un inicio, la crisis fue tomada como la oportunidad perfecta de exigirles a figuras comunistas, como Diego Rivera y David Alfaro Siqueiros, un posicionamiento claro y reafirmar el respaldo de la intelectualidad mexicana para la causa anticomunista. Sin embargo, y a pesar de los esfuerzos de García Treviño, importantes defensores del CLC, como Rómulo Gallegos, Alfonso Reyes, Salvador Azuela y Pedro de Alba, se negaron a suscribir una posición antisoviética. En palabras de los dirigentes locales del CLC, esto se debió principalmente al miedo de estas figuras a "quedar mal con (Lázaro) Cárdenas", quien en 1955 había recibido el Premio Stalin de la Paz y había firmado una "declaración rusófila” respecto al problema en Hungría. ${ }^{43}$

En ambos países, el anticomunismo del Congreso por la Libertad de la Cultura encontró un importante freno en el antiimperialismo de importantes sectores de las elites intelectuales. En el caso de México, es evidente que la

40 The Indian Committee and Hungary, Report. IACFR, caja 58, exp. 3.

41 Jayaprakash Narayan, "Nehru's Stand on Hungary, Bombay, 11 November 1956", citado en Prasad (2005, pp. 150-152).

${ }^{42}$ Carta de Eric Da Costa a Nicolas Nabokov, 21 de noviembre de 1956; Carta de Nicolas Nabokov a Eric Da Costa, 29 de noviembre de 1956. IACFr, caja 122, exp. 7.

43 Telegrama de Julián Gorkin a Rodrigo García Treviño, 7 de noviembre de 1956; Carta de Rodrigo García Treviño a Julián Gorkin, 13 de diciembre de 1956. IACFR, caja 216, exp. 5a. 
fuerza de las posturas antiestadunidenses -que alimentaron perplejidad de figuras como Gorkin, formado en la confrontación entre comunismo estalinista y socialismo anticomunista de la Europa de entreguerras- chocaron de manera frontal con la aceptación de las cabecillas del CLC respecto a la política exterior de Estados Unidos en Latinoamérica. Esto contrastaba también con el creciente conservadurismo de la sociedad mexicana de las décadas del "milagro" y alimentaba la ambigua postura de México en el contencioso escenario de la guerra fría latinoamericana. Al mismo tiempo, es claro que para los defensores mexicanos de la libertad de la cultura los sucesos que tenían lugar en Cuba o Guatemala resultaban mucho más urgentes que los conflictos en China o Hungría. En contraste, en India, los seguidores del cLC sí montaron un frente de abierta oposición a las políticas y posturas geopolíticas del Estado nehruviano, como queda claro en el posicionamiento de figuras como Narayan ante la crisis en Hungría.

Siguiendo el impulso y el reconocimiento público ganado tras la crisis de Hungría, el cLc en India se constituyó como un foro para el activismo político en torno a la ocupación China del Tíbet. A principios de 1959, una importante crisis diplomática estalló entre China e India después de que miles de tibetanos encabezados por el Dalai Lama, que cruzaran la frontera desde China hacia India escapando de la violenta represión del alzamiento de Lhasa de marzo de aquel año, fueran recibidos como refugiados por el gobierno de India. Al poco tiempo, importantes miembros del CLC en India comenzaron a preparar la impresión de 4000 ejemplares de la antología Tíbet lucha por su libertad, la cual fue enviada a contactos del Congreso en todo el mundo. ${ }^{44} \mathrm{La}$ publicación, que contó con una introducción escrita por Jayaprakash Narayan y un prólogo del Dalai Lama, fue también publicada en español, bahasa indonesio y árabe, lo que le permitió tener una amplia circulación en el Tercer Mundo. ${ }^{45}$ Este temprano análisis sobre la crisis del Tíbet -quizá la primera en ser leída por el público hispanohablante de Latinoamérica- estaba enmarcado por la lógica militante y anticomunista de los seguidores del CLC. Para Naraya, la ocupación del Tíbet representaba "una de las más grandes tragedias de la historia" y la prueba rotunda de que el comunismo represenexp. 4.

${ }^{44}$ Carte de Prabhakar Padhye a Jorgen Schleimann, 3 de abril de 1959. IACFr, caja 180,

${ }_{45}$ Carta de V. B. Karnik a John C. Hunt, 6 de septiembre de 1960. IAcFr, caja 508, exp. 10. 
taba una nueva y peligrosa forma de imperialismo. ${ }^{46}$ Este acontecimiento sirvió para activar las redes del CLC en India, donde en mayo de 1959 se celebró la Convención India por el Tíbet, que reunió a casi 2000 representantes de numerosos países de Asia ${ }^{47}$ y sirvió de plataforma para la creación del Comité Afro-Asiático por el Tíbet. Este último, que tuvo una aparición breve en la Asamblea de las Naciones Unidas, reunió a representantes de Asia y África -incluyendo a intelectuales como el senegalés Leopold Senghor- y representó la cúspide del activismo internacional de la sede India del Congreso por la Libertad de la Cultura. ${ }^{48}$

\section{COMENTARIOS FINALES}

A medida que la década de 1960 avanzaba y la confrontación ideológica de la guerra fría se hacía más violenta, importantes sectores de la intelligentsia en el Tercer Mundo comenzaron a mostrarse cada vez más recelosos del intervencionismo cultural estadunidense. En el caso de México, la revolución cubana y la evidente coincidencia entre el anticomunismo del Atlántico Norte y las posturas represivas del régimen del PRI abrieron nuevos espacios para el fortalecimiento de la crítica de izquierda y el antiyankismo tradicional de amplios sectores de la población. En India, el desvanecimiento del entusiasmo generado por la independencia de 1947 se mezcló con la alarma generada por el intervencionismo estadunidense en Vietnam para dar paso a un claro y vehemente rechazo de la política internacional y cultural de Estados Unidos en Asia. Finalmente, las escandalosas revelaciones periodísticas de 1967 precipitaron el eclipse del Congreso por la Libertad de la Cultura y anunciaron el inicio de una nueva etapa en la guerra fría cultural marcada por el descrédito creciente de las iniciativas internacionales de Estados Unidos en el

${ }^{46}$ Forum-Service, 1959. IACFR, caja 179, exp. 7.

47 "Friends of Congress Active in Behalf of Tibet", Congress News for Cultural Freedom, mayo-junio de 1959, 2. Michael Josselson Papers, caja 27, exp. 6. Harry Ransom Center, The University of Texas at Austin.

${ }^{48}$ Carta de Leela P. Trikamdas a Armand Gaspar, 23 de marzo de 1962. IACFR, caja 174, exp. 8; Sumary of a report on Tibet: submitted to the International Commission of Jurists by Shri Purshottam Trikamdas (5 de junio de 1959), International Commission of Jurists. https:// www.icj.org/summary-of-a-report-on-tibet-submitted-to-the-international-commission-of-jurists-by-shri-purshottam-trikamdas-senior-advocate-supreme-court-of-india/ [consulta: 2 de septiembre de 2018]. 
Tercer Mundo y una polarización que desbordó los parámetros pretendidamente "post-ideológicos" de la década de 1950.

A pesar de este estrepitoso declive, y como hemos visto en estas páginas, durante poco más de una década y media el Congreso por la Libertad de la Cultura funcionó como un importante foro de debate y activismo cultural que marcó profundamente las trayectorias del debate intelectual en India y México durante las décadas siguientes. Por un lado, el cLc facilitó la consolidación de una esfera de actividad intelectual al margen del Estado y encabezada por la figura del empresario de la cultura -encarnado por figuras como Gorkin, Masani y García Treviño- desde la que se promovieron posicionamientos ideológicos e intelectuales relacionados con el liberalismo postutópico y anticomunista de pensadores como Edward Shils, Daniel Bell, Seymour Lipset y W. W. Rostow. Estas ideas, asociadas con el principio del antitotalitarismo y el ideal de la modernización, sentaron las bases para un nuevo dogma que definió los contornos de gran parte de la discusión académica, pública y política encabezada por las nuevas elites del Tercer Mundo a partir de la década de 1970 y abrieron las puertas a la aceptación global de nuevas formas de gobernanza y análisis social relacionadas en décadas recientes con el neoliberalismo. En este sentido, la fugaz y accidentada historia del Congreso por la Libertad de la Cultura en India y México nos ofrece muchas importantes pistas para entender la historia intelectual, ideológica y política de la segunda mitad del siglo xx en dos regiones centrales del Tercer Mundo, así como para descifrar las trayectorias que unieron al entramado transcontinental de intercambio tejido desde el Atlántico Norte durante las primeras décadas de la guerra fría y los profundos cambios culturales y políticos vividos en regiones como Asia y Latinaomérica a partir de la década de 1970.

\section{LISTA DE REFERENCIAS}

Alburquerque, G. (2011). La trinchera letrada. Intelectuales latinoamericanos y guerra fría. Santiago: Ariadna Ediciones.

Amat, J. (2016). La semilla del liberalismo. Política y literatura en torno a la actividad española del Congreso por la Libertad de la Cultura (1958-1969). (Tesis de doctorado inédita). Universidad de Barcelona, España.

Anderson, K. B. (2010). Marx at the margins: On nationalism, ethnicity, and non-western societies. Chicago: The University of Chicago Press. 
Anderson, A. (2011). Character and ideology: The case of cold war liberalism. New Literary History, 42(2), 209-229. DoI: https://doi.org/10.1353/nlh.2011.0017

Bell, D. (1960). The end of ideology: On the exhaustion of political ideas in the fifties. Nueva York: Free Press.

Berlin, I. (1990). The crooked timber of humanity. Princeton, New Jersey: Princeton University Press.

Burke, R. (2016). "Real problems to discuss": The Congress for Cultural Freedom's Asian and African expeditions, 1951-1959. Journal of World History, 27(1), 53-85. DoI: https://doi.org/10.1353/jwh.2016.006

Chakrabarty, D. (2005). Legacies of bandung: Decolonisation and the politics of culture. Economic and Political Weekly, 40(46), 4812-4818. Recuperado de http://www. jstor.org/stable/4417389

Chaudhuri, R. (2013). Forged in crisis: India and the United States since 1947. Londres: C. Hurst \& Co.

Dube, S. C. (1958). India's changing villages: Human factors in community development. Londres: Routledge and Kegan Paul Ltd.

Cohn, D. (2005). The Mexican intelligentsia, 1950-1968: Cosmopolitanism, national identity, and the State. Mexican Studies/Estudios Mexicanos, 21(1), 141-182. DoI: https://doi.org/10.1525/msem.2005.21.1.141

Coleman, P. (1989). The liberal conspiracy. The Congress for Cultural Freedom and the struggle for the mind of postwar Europe. Nueva York: Free Press.

Franco, J. (2002). The decline and fall of the lettered city. Latin America in the cold war. Cambridge, Mass.: Harvard University Press.

Gilman, N. (2003). Mandarins of the future. Modernization theory in cold war America. Baltimore: Johns Hopkins University Press.

Hegel, F. W. G. (1956). Philosophy of history. Nueva York: Dover.

Holt, E. M. (2013). "Bread or freedom”: The Congress for Cultural Freedom, the CIA, and the Arabic Literary Journal Hiwār (1962-67). Journal of Arabic Literature, 44(1), 83-102. DoI: https://doi.org/10.1163/1570064x-12341257

Iber, P. (2015). Neither peace nor freedom: The cultural cold war in Latin America. Cambridge, Mass.: Harvard University Press.

Indian Congress for Cultural Freedom (1951). Indian Congress for Cultural Freedom. March 28 to 31, 1951. Bombay: Kanada Press.

Glondys, O. (2018). Dismissals of the Congress for Cultural Freedom's representatives in Latin America as part of the strategy of "Opening to the left" (19611964). Culture and History Digital Journal, 7(1), 87-98. Dor: https://doi.org/10.3989/ chdj.2018.010 
Jannello, K. (2012). El Congreso por la Libertad de la Cultura: el caso chileno y la disputa por las "ideas de fuerza" de la guerra fría. Revista Izquierdas, 14, 14-52. Recuperado de http://www.izquierdas.cl/ediciones/2012/numero-14-diciembre

Jannello, K. (2014). Redes intelectuales y guerra fría: la agenda argentina del Congreso por la Libertad de la Cultura. Revista de la Red Intercátedras de Historia de América Latina Contemporánea, 1, 60-85. Recuperado de https://revistas.unc.edu. ar/index.php/RIHALC/article/view/8365

Keller, R. (2015). Mexico's cold war. Cuba, the United States, and the legacy of the Mexican revolution. Nueva York: Cambridge University Press.

Kent Carrasco, D. (2016). Jayaprakash Narayan and Lok Niti: socialism, gandhism and political cultures of protest in Xx century India. (Tesis de doctorado indédita). King's College, Londres, Reino Unido.

Kent Carrasco, D. (2020). El socialismo Gandhiano. Crítica a la modernidad y utopía poscolonial. En C. Illades, R. Mondragón y F. Quijano (eds.), En ningún lugar y en todas partes. Utopía y socialismo, un horizonte compartido (pp. 283-306). México: Universidad Nacional Autónoma de México.

Latham, R. (1997). The liberal moment. Modernity, security, and the making of the postwar international order. Nueva York: Columbia University Press.

Latham, M. E. (2003). Modernization as ideology. American social science and "Nation Building" in the Kennedy era. Chapel Hill: The University of North Carolina Press.

Lewis, S. L. (2019). Asian socialism and the forgotten architects of post-colonial freedom, 1952-1956. The Journal of World History, 30(1-2), 55-88. Dor: https://doi. org/10.1353/jwh.2019.0013

Loaeza, S. (2006). La fractura mexicana y el golpe de 1954 en Guatemala. Historia Mexicana, 66(2), 725-791. DoI: http://dx.doi.org/10.24201/hm.v66i2.3346

Masani, M. R. (1955). The mind of Asia. Foreign Affairs. Recuperado de https://www. foreignaffairs.com/articles/east-asia/1955-07-01/mind-asia

McGarr, P. M. (2013). The cold war in South Asia: Britain, the United States and the Indian subcontinent, 1945-1965. Cambridge: Cambridge University Press.

Medina, L. (1979). Historia de la Revolución Mexicana, 1940-1952: civilismo y modernización del autoritarismo. Distrito Federal: El Colegio de México.

Metcalfe, T. R. (1997). Ideologies of the raj. Nueva York: Cambridge University Press.

Moreno, J. (2004). Yankee don't go home!: Mexican nationalism, American business culture, and the shaping of modern Mexico, 1920-1950. Chapel Hill, N. C.: University of North Carolina Press.

Nanda, B. R (1998). Nehru and non-alignment. Delhi: Oxford University Press. 
Nayudu, S. K. (2015). The Nehru years: Indian non-alignment as the critique, discourse and practice of security (1947-1964). (Tesis de doctorado inédita). King's College, Londres, Reino Unido.

Nehru, J. (2002). Speech inaugurating the new building of the Punjab High Court, Chandigarh, March 19, 1955. En Selected works of Jawaharlal Nehru (vol. 28, 1 February-31 May 1955). Nueva Delhi: Oxford University Press.

Prasad, B. (ed.) (2005). Jayaprakash Narayan. Selected works (vol. viI, 1954-1960). Delhi: Manohar.

Pullin, E. D. (2011). "Money does not make any difference to the opinions that we hold": India, the CIA, and Congress for Cultural Freedom. Intelligence and National Security, 26(2-3), 377-98. DoI: https://doi.org/10.1080/02684527.2011.559325

Reid, E. (1981). Envoy to Nehru. Nueva York: Oxford University Press.

Rivera Mir, S. (2018). La difusión del marxismo en tiempos convulsos. Rodrigo García Treviño y Editorial América (1936-1940). En A. Granados y S. Rivera Mir (eds.), Prácticas editoriales y cultura impresa entre los intelectuales latinoamericanos del siglo $\mathrm{XX}$ (pp. 71-98). Zinacantepec-Ciudad de México: El Colegio Mexiquense/Universidad Autónoma Metropolitana.

Roosevelt, E. (1954). India and the awakening east. Londres: Hutchinson.

Rubin, A. N. (2012). Archives of authority. Empire, culture and the cold war. Princeton, N. J.: Princeton University Press.

Santos, E. (1956). Presentación. Cuadernos, 19.

Saunders, F. S. (2001). La cia y la guerra fría cultural. Barcelona: Debate.

Schlesinger jr., A. M. (2017). The vital center. The politics of freedom. Londres y Nueva York: Rutledge.

Shils, E. (1958). Ideology and civility: on the politics of the intellectual. The Sewanee Review, 66(3), 450-480.

Shils, E. (septiembre, 1990). Remembering the Congress for Cultural Freedom. Encounter, 52-65.

Scott-Smith, G. (2000). "A radical democratic political offensive": Melvin J. Lasky, Der Monat, and the Congress for Cultural Freedom. Journal of Contemporary History, 35(2), 263-280. Dor: https://doi.org/10.1177/002200940003500207

Scott-Smith, G. (2001). The politics of apolitical culture. The Congress for Cultural Freedom and the political economy of American hegemony 1945-1955. Londres: Routledge.

Scott-Smith, G. (2002). The Congress for Cultural Freedom, the end of ideology, and the 1955 Milan conference: "Defining the parameters of discourse". Journal of Contemporary History, 37(3), 437-455. DOI: https://doi.org/10.1177/0022009402037003 0601 
Trilling, L. (2008). The liberal imagination (e-book). Nueva York: New York Review of Books.

Paz, O. (1993). El laberinto de la soledad. Madrid: Cátedra.

Ruiz Galbete, M. (2018). ¿"Fidelismo sin Fidel”? El Congreso por la Libertad de la Cultura y la Revolución Cubana. Historia Crítica, 67, 111-132. Dor: https://doi. org/10.7440/histcrit67.2018.06

Sen, T. (2017). India, China, and the world. A connected history. Nueva Delhi: Oxford University Press.

Westad, O. A. (2005). The global cold war. Third world interventions and the making of our times. Nueva York: Cambridge University Press.

\section{OTRAS FUENTES}

\section{Archivos}

Harry Ransom Center, The University of Texas at Austin.

IACFR International Association for Cultural Freedom Records. Special Collections. The University of Chicago Library. 